\title{
AVALIAÇÃO DO PERFIL SENSORIAL E TESTE DE CONSUMIDOR DE BATATA PALHA
}

\author{
BIBIANA ALVES DOS SANTOS* \\ RAQUEL FORMIGHIERI** \\ ADRIELE MARTINS** \\ MARIA CECÍLIA ENES RIBEIRO* \\ DIOGO MAUS* \\ ANA KAROLINE FERREIRA IGNÁCIO** \\ LARISSA FONTANA*** \\ NOELIA BEDOYA** \\ APARECIDO PAULA JUNIOR**

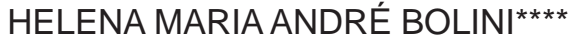

\begin{abstract}
O objetivo deste estudo foi determinar o perfil sensorial, a aceitação e a intenção de compra de seis marcas comerciais de batata palha tradicional, denominadas A, B, C, D, E e F, adquiridas em supermercados da região de Campinas (SP). Pela Análise Descritiva Quantitativa (ADQ) foram escolhidos 13 termos descritores para batata-palha: cor amarela e queimada, presença de sal na superfície, espessura do corte, aromas de batatafrita, ranço e queimado, sabor salgado, queimado, de ranço e de batata-palha frita, textura, crocância e oleosidade. Os resultados da ANOVA do teste afetivo mostraram que os atributos que mais influenciaram os consumidores foram cor, sabor e textura, sendo as maiores médias encontradas para as marcas $D$ e $E$. A marca $F$ diferiu significativamente $(p<0,05)$ em relação às demais, com valores superiores nos parâmetros cor amarela, queimada e sabor de ranço e queimado. Os componentes principais 1, cor amarela e 2 , textura crocante explicaram juntos $85,85 \%$ da variação entre as marcas. No mapa da correlação da regressão PLS da impressão global dos consumidores e características das amostras destacouse a proximidade no gráfico do atributo crocância em relação à impressão global, o que sinaliza a preferência do consumidor por batatas palha com maior crocância.
\end{abstract}

PALAVRAS-CHAVE: BATATA PALHA; PERFIL SENSORIAL DESCRITIVO; TESTE AFETIVO.

* $\quad$ Doutorandos em Tecnologia de Alimentos, Faculdade de Engenharia de Alimentos (FEA), Universidade Estadual de Campinas (UNICAMP), Campinas, SP, Brasil (e-mail: bialvesantos@yahoo.com.br; cissaribeiro@gmail.com; diogo.maus@gmail.com).

** Mestres em Tecnologia de Alimentos, FEA, UNICAMP, Campinas, SP, Brasil (e-mail: quel_f7@hotmail. com; adriele.martins@hotmail.com; anakarolineign@gmail.com; noelia.bedoya@gmail.com; apjr@fea. unicamp.br).

*** Especialista em Alimentos, Professora, Curso Técnico em Nutrição e Dietética, Centro Estadual de Educação Tecnológica Paula Souza - ETEC Prefeito Aberto Feres, Araras, SP, Brasil (e-mail: lari-fontana@ hotmail.com).

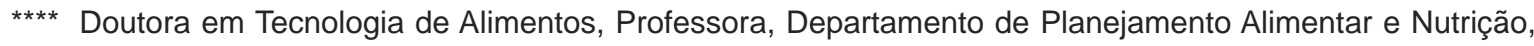
FEA, UNICAMP, Campinas, SP, Brasil (e-mail: hellini@fea.unicamp.br). 


\section{INTRODUÇÃo}

A batata (Solanum tuberosum L.) constitui um dos alimentos mais consumidos no mundo, devido à sua composição, versatilidade gastronômica e tecnológica, assim como pelo baixo preço dos tubérculos (COELHO, VILELA e CHAGAS, 1999, FREITAS et al., 2006). A comercialização de batatas sempre ocorreu em sua maior parte na forma "in natura", porém sua industrialização vem aumentando em todas as partes do mundo, destacando-se os produtos tipo "chips" e batatas fritas congeladas, devido à facilidade e rapidez no preparo (FREITAS et al.,2006; RODRIGUES, 1990; ARRUDA, 2004). A batata tornou-se a hortaliça de maior importância econômica no Brasil, sendo a fritura a forma preferencial de preparo comercial e doméstica (ZORZELLA et al., 2003).

Produtos que podem ser consumidos diretamente, como "chips" ou batata-palha, ou prontos para serem preparados (como batatas descascadas e cortadas em palitos resfriados e préfritos congelados) têm alcançado cada vez mais espaço no mercado, em vários países, incluindo o Brasil (FREITAS et al., 2006). Destaca-se também o fator custo, já que a indústria pode oferecer o produto a preços competitivos, devido à possibilidade de adquirir grandes volumes e aproveitar os subprodutos de processamento (RODRIGUES, 1990).

De acordo com a Associação Brasileira de Batata (ABBA, 2009), as principais variedades de batata cultivadas no Brasil são: Ágata, Asterix, Bintje, Monalisa, Mondial e Atlantic. As variedades Asterix, Atlantic e Bintje são as mais indicadas para fritura devido, entre outros fatores, ao alto teor de matéria seca.

As batatas-palha são pequenos filetes de batata, fritos por imersão em óleo ou gordura hidrogenada de origem vegetal. Os produtos alimentícios que mais se assemelham às batataspalha são os chips, os quais apresentam características de textura, cor e sabor muito similares. A oferta de batata-palha nos supermercados aumentou consideravelmente e grandes empresas do ramo alimentício que por muito tempo só processavam batata sob a forma de chips passaram também a processar batata-palha (REIS, 2007).

Os parâmetros mais relevantes na avaliação sensorial de batatas palha ou chips são a cor, o aroma, o sabor e a crocância. É desejável que as batatas fritas apresentem coloração dourada clara, sem chegar ao marrom e ausência de pontos ou traços escuros (marrons e esverdeados). Os teores de matéria seca e açúcares redutores assumem grande importância em relação ao rendimento e qualidade dos produtos processados, determinando a absorção de gordura durante a fritura, a textura e o sabor do produto final (CAPEZIO, HUARTE e CARROZZI, 1993). Outro fator que pode alterar consideravelmente a qualidade das batatas fritas envolve o teor residual de óleo. Alto teor residual de óleo no produto final aumenta os custos de produção e prejudica sua crocância e sabor. Por outro lado, baixos teores resultam em perda do aroma e do sabor característicos de produtos fritos (TFOUNI et al., 2003).

As batatas palhas ou chips não devem apresentar amargor ou sabores indesejáveis como o de queimado. A qualidade do sabor depende principalmente das matérias-primas (batata e óleo) e do processamento adequado. Já a crocância do produto final está relacionada à umidade da batata e à temperatura e tempo de fritura. As batatas com alto teor de umidade absorvem mais óleo durante a fritura, o que as torna murchas. A crocância também é afetada pela espessura dos filetes de batata (TFOUNI et al., 2003).

Para avaliar todas as características sensoriais de batatas palha ou chips, os métodos descritivos, tais como Perfil de Sabor, Perfil de Textura e Análise Descritiva Quantitativa (ADQ) são de grande utilidade. Tais métodos auxiliam a solução de diversos problemas associados ao controle de qualidade, estudos de vida útil, desenvolvimento de novos produtos ou a interpretação das preferências dos consumidores.

O método da Análise Descritiva Quantitativa (STONE, BLEIBAUM e THOMAS, 2012) é muito utilizado para traçar da forma mais completa possível, o perfil sensorial dos produtos comercializados e desenvolvidos em diversos estudos. Por meio dos atributos aparência, odor, textura e sabor buscase a identificação e quantificação de todas as características encontradas no produto avaliado. 
Devido ao crescimento do consumo de batatas palha no Brasil, este estudo teve como objetivos traçar o perfil sensorial de seis amostras comerciais de batata-palha mediante Análise Descritiva Quantitativa (ADQ) e avaliar sensorialmente a aceitação e a atitude de compra do consumidor em relação às amostras de batata-palha analisadas.

\section{MATERIAL E MÉTODOS}

\subsection{MATERIAL}

Foram avaliadas seis amostras comerciais de batata palha, adquiridas em supermercados da região de Campinas (SP), sendo todas as batatas palha do tipo tradicional. As amostras de batata palha foram identificadas pelos códigos A, B, C, D, E e F. Os testes sensoriais foram realizados no Laboratório de Análise Sensorial da Faculdade de Engenharia de Alimentos da Universidade Estadual de Campinas (UNICAMP), cujas instalações incluem cabines individuais, controle de iluminação e de odores e temperatura ambiente.

Determinou-se o perfil sensorial de cada amostra utilizando a Análise Descritiva Quantitativa (ADQ) descrita por Stone, Bleibaum e Thomas (2012), com 9 julgadores treinados. Realizou-se ainda o teste de aceitação e intenção de compra com a participação de consumidores do produto, conforme recomendações de Meilgaard, Civille e Carr (2006).

\subsection{ANÁLISE SENSORIAL}

\subsubsection{Análise Descritiva Quantitativa}

Os julgadores treinados realizaram o levantamento dos termos descritores sensoriais da batata palha pelo método tradicional de análise descritiva quantitativa (STONE, BLEIBAUM e THOMAS, 2012), sendo as amostras apresentadas aos julgadores de forma monádica e em cabines individuais de avaliação sensorial. Os julgadores foram solicitados a avaliar as amostras, utilizando a Ficha de Aplicação do Método de Análise Descritiva Quantitativa (Figura 1).

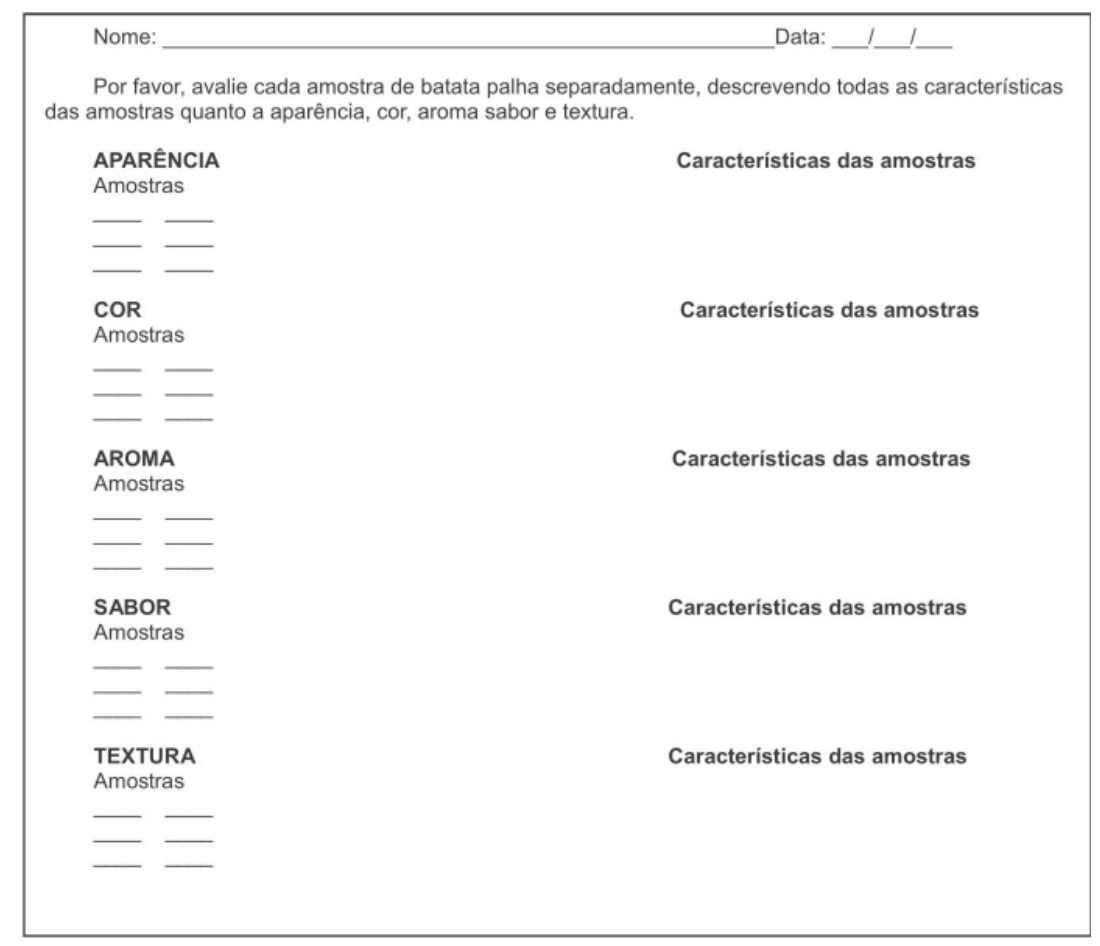

FIGURA 1 - FICHA DE APLICAÇÃO DO MÉTODO DE ANÁLISE DESCRITIVA QUANTITATIVA TRADICIONAL 
Após cada julgador ter gerado seus próprios termos para descrever todas as características de cada amostra ocorreu a discussão em grupo com o objetivo de reunir os termos descritivos semelhantes e gerar amostras de referência. Com os termos descritores gerados elaborou-se a ficha de avaliação, utilizando escalas não estruturadas de $9 \mathrm{~cm}$, conforme demonstrado na Figura 2.

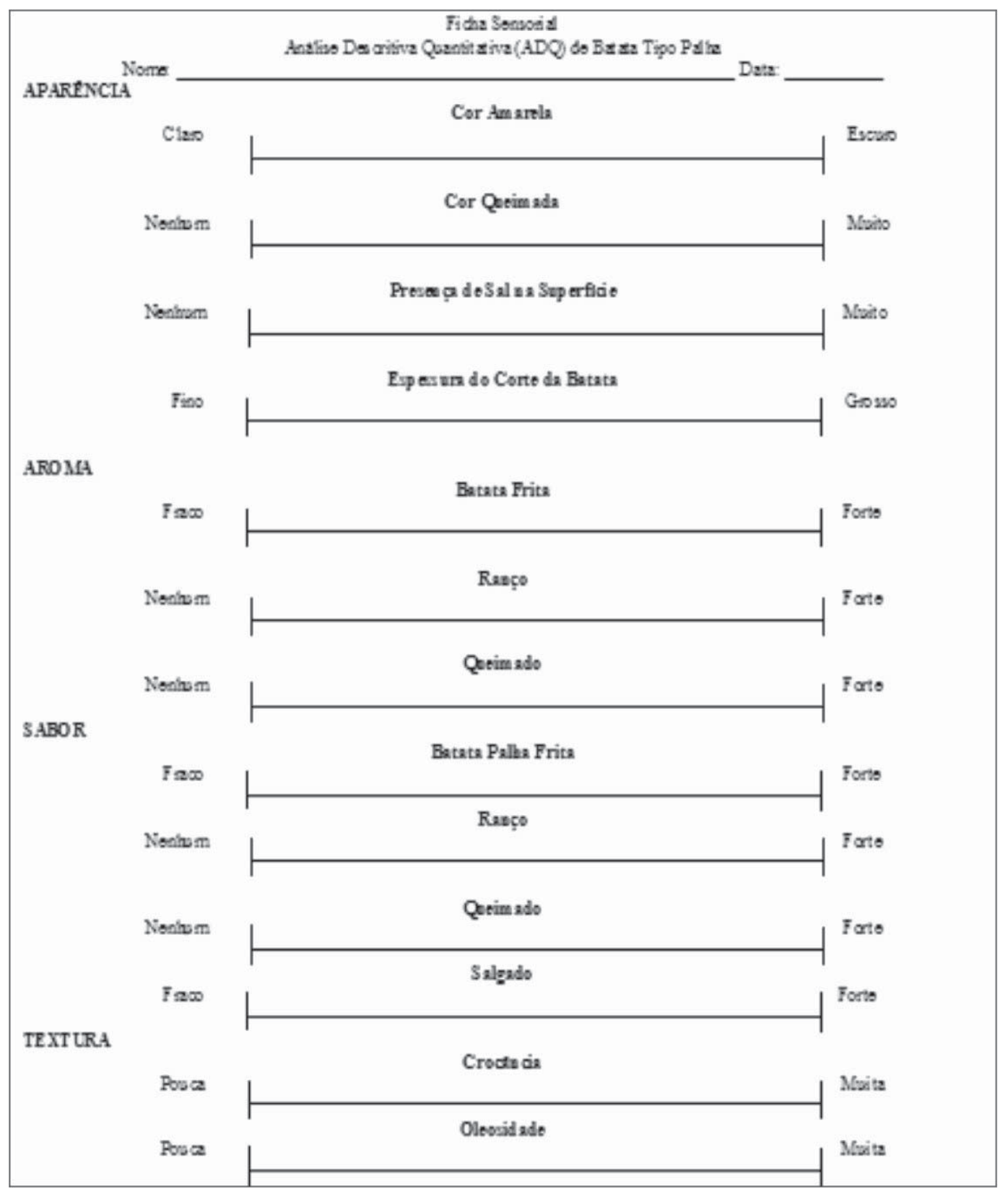

\section{FIGURA 2 - FICHA DE ANÁLISE SENSORIAL COM ESCALA NÃO ESTRUTURADA UTILIZADA PARA A ADQ}

Selecionou-se a equipe definitiva para a análise descritiva quantitativa mediante testes, utilizando a ficha elaborada (Figura 2) com as escalas de intensidade para os termos definidos. Foram selecionados os candidatos com base no poder de discriminação entre amostras, repetibilidade e concordância entre os julgadores (DAMÁSIO e COSTEL, 1991), verificada por análise de variância de dois fatores ( $F$ amostra e $F$ repetição) para cada julgador em relação a cada atributo (STONE, BLEIBAUM e THOMAS, 2012). A equipe selecionada de 9 julgadores apresentou valores de $F$ amostra significativa para $p<0,30$ e $F$ repetição não significativa para $p>0,05$ e concordância das médias da equipe. 
Os julgadores selecionados avaliaram as seis amostras de batata palha realizando três repetições, com auxílio da ficha definitiva para o teste mostrada na Figura 2. As amostras foram apresentadas codificadas com algarismos de três dígitos, em blocos completos balanceados de forma monádica (STONE, BLEIBAUM e THOMAS, 2012) em cabines individuais do Laboratório de Análise Sensorial.

\subsubsection{Teste afetivo de aceitação e atitude de compra}

Realizou-se teste afetivo (teste de consumidor), no qual todas as amostras de batatapalha foram avaliadas por 128 consumidores do produto em relação à cor, aroma, sabor, textura e impressão global.

As amostras foram servidas em blocos completos balanceados, de forma monádica, em copos plásticos codificados com algarismos de três dígitos. Utilizou-se escala hedônica não estruturada de $9 \mathrm{~cm}$, com extremos que variaram de "desgostei muitíssimo a "gostei muitíssimo" (STONE, BLEIBAUM e THOMAS 1993).

A atitude dos consumidores em relação à compra do produto também foi avaliada, mediante escala de intenção de compra de cinco pontos, que variou de "certamente compraria" a "certamente não compraria" (MEILGAARD, CIVILLE e CARR, 2006).

O teste de aceitação e de atitude de compra foi realizado em cabines individuais do Laboratório de Análise Sensorial da UNICAMP.

\subsubsection{Análise estatística}

Adotou-se delineamento experimental de blocos completos em relação aos julgadores e blocos completos balanceados em relação às amostras que foram apresentadas de forma monádica.

Os resultados da ADQ foram submetidos à Análise de Variância (ANOVA), Teste de Tukey ao nível de $5 \%$ de significância para a comparação entre as médias e Análise de Componentes Principais. Os resultados do teste de aceitação foram analisados por meio de Análise de Variância e teste de Tukey para médias, também ao nível de $5 \%$ de significância $(p \leq 0,05)$. Todas as análises estatísticas foram realizadas utilizando-se os programas do pacote estatístico SAS (2003).

A correlação dos resultados da análise dos atributos sensoriais com os resultados da aceitação pelos consumidores foi efetuada pela análise estatística multivariada de regressão por quadrados mínimos parciais (partial least square regression - PLSR), usandose o software estatístico XLSTAT-MX (2005). Os resultados das médias foram apresentados de forma tabular e gráfica e a análise dos componentes principais mediante gráficos bidimensionais.

\section{RESULTADOS E DISCUSSÃO}

Nove julgadores participaram do levantamento dos termos descritores, definidos por consenso pelo grupo e os atributos selecionados são apresentados na Tabela 1, com suas respectivas referências, conforme o Método de Rede - Kelly's Reportory Grid Method (MURRAY, DELAHUNTY e BAXTER, 2001).

As seleções dos principais atributos para avaliação do perfil sensorial de batatas palha concordaram com os termos descritivos encontrados por Almeida et al., 1983. 

TABELA 1 - DEFINIÇÕES E REFERÊNCIAS PARA OS TERMOS DESCRITORES DAS
AMOSTRAS DE BATATA-PALHA LEVANTADOS PELOS JULGADORES

\begin{tabular}{ccc}
\hline $\begin{array}{c}\text { Termo Descritor } \\
\text { (Atributo) }\end{array}$ & Definição & Referências \\
\hline & Aparência \\
\hline
\end{tabular}

Cor amarela (CAM) Característica da gema de ovo

Cor queimada (CQU)

Característica de acúcar caramelizado

Presença de sal na superfície da batata (SSU)

Presença de grânulos de sal na superfície da batata visíveis ao olho nu

Claro: margarina extra cremosa com sal da marca Doriana ${ }^{\circ}$.

Escuro: biscoito tipo Cream Cracker da marca Tostines ${ }^{\circledR}$

Nenhum: $60 \mathrm{~g}$ de batata frita em $200 \mathrm{~mL}$ de óleo de soja da Marca Sadia ${ }^{\circledR}$ à temperatura de $200^{\circ} \mathrm{C}$ por 1 $\min$.

Muito: $60 \mathrm{~g}$ de batata frita em $200 \mathrm{~mL}$ de óleo de soja da Marca Sadia ${ }^{\circledR}$ à temperatura de $200^{\circ} \mathrm{C}$ por $7 \mathrm{~min}$

Nenhum: $60 \mathrm{~g}$ de batata frita em $200 \mathrm{~mL}$ de óleo de soja da Marca Sadia ${ }^{\circledR}$ à temperatura de $200^{\circ} \mathrm{C}$ por 4 min, sem nenhuma adição de sal após a fritura

Muito: $60 \mathrm{~g}$ de batata frita em $200 \mathrm{~mL}$ de óleo de soja da Marca Sadia ${ }^{\circledR}$ à temperatura de $200^{\circ} \mathrm{C}$ por $4 \mathrm{~min}$, com adição de 5\% de sal após a fritura

Fino: grafite $0.9 \mathrm{~mm}$ da marca Faber Castell $®$

Grosso: palito de fósforo marca Fiat Lux (Pinheiro)®

Aroma

Espessura do corte da Largura em que as batatas palhas estão cortadas

Característico de batata

Batata Frita (ABP) frita recentemente em óleo de soja da marca Sadia ${ }^{\circledR}$

Cheiro forte e acre que o contato com o ar produz em substâncias gordurosas

Ranço (ARN)

Cheiro próprio daquilo que assou ou torrou demais

Batata Palha Frita (SBP)

Característico de batata frita recentemente em óleo de soja da marca Sadia ${ }^{\circledR}$

Conjunto de sensações

Ranço (SRN) de gosto e aroma fortes e acres das substâncias gordurosas
Fraco: $60 \mathrm{~g}$ de batata frita em $200 \mathrm{~mL}$ de óleo de soja da Marca Sadia ${ }^{\circledR}$ à temperatura de $200^{\circ} \mathrm{C}$ por $1 \mathrm{~min}$ Forte: $60 \mathrm{~g}$ de batata frita em $200 \mathrm{~mL}$ de óleo de soja da Marca Sadia ${ }^{\circledR}$ à temperatura de $200^{\circ} \mathrm{C}$ por 4 min

Nenhum: $100 \mathrm{~mL}$ de óleo de soja da marca Sadia ${ }^{\circledR}$

Forte: $100 \mathrm{~mL}$ de óleo de fritura estocado por 4 meses

Nenhum: $60 \mathrm{~g}$ de batata frita em $200 \mathrm{~mL}$ de óleo de soja da Marca Sadia ${ }^{\circledR}$ à temperatura de $200^{\circ} \mathrm{C}$ por 4 $\min$

Muito: $60 \mathrm{~g}$ de batata frita em $200 \mathrm{~mL}$ de óleo de soja da Marca Sadia ${ }^{\circledR}$ à temperatura de $200^{\circ} \mathrm{C}$ por $7 \mathrm{~min}$

\section{Sabor}

Fraco: branqueamento da batata em água fervente por $30 \mathrm{~s}, 60 \mathrm{~g}$ de batata palha frita em $200 \mathrm{~mL}$ de óleo de soja da Marca Sadia ${ }^{\circledR}$ à temperatura de $200^{\circ} \mathrm{C}$ por 4 min

Forte: $60 \mathrm{~g}$ de batata palha frita em $200 \mathrm{~mL}$ de óleo de soja da Marca Sadia ${ }^{\circledR}$ à temperatura de $200^{\circ} \mathrm{C}$ por 5 min

Nenhum: $60 \mathrm{~g}$ de batata palha frita em $200 \mathrm{~mL}$ de óleo de soja da Marca Sadia ${ }^{\circledR}$ à temperatura de $200^{\circ} \mathrm{C}$ por $4 \mathrm{~min}$

Forte: $60 \mathrm{~g}$ de batata palha frita em $200 \mathrm{~mL}$ de óleo de fritura estocado por período de 4 meses à temperatura de $200^{\circ} \mathrm{C}$ por $3 \mathrm{~min}$ 


\begin{tabular}{|c|c|c|}
\hline $\begin{array}{l}\text { Termo Descritor } \\
\text { (Atributo) }\end{array}$ & Definição & Referências \\
\hline \multicolumn{3}{|c|}{ Sabor } \\
\hline Queimado (SQU) & $\begin{array}{l}\text { Sabor próprio daquilo que } \\
\text { assou ou torrou demais }\end{array}$ & $\begin{array}{l}\text { Nenhum: } 60 \mathrm{~g} \text { de batata frita em } 200 \mathrm{~mL} \text { de óleo de } \\
\text { soja da Marca Sadia }{ }^{\circledR} \text { à temperatura de } 200^{\circ} \mathrm{C} \text { por } \\
4 \text { min } \\
\text { Muito: } 60 \mathrm{~g} \text { de batata frita em } 200 \mathrm{~mL} \text { de óleo de soja } \\
\text { da Marca Sadia } ₫ \text { à temperatura de } 200^{\circ} \mathrm{C} \text { por } 7 \text { min }\end{array}$ \\
\hline Salgado (SSA) & $\begin{array}{l}\text { Sabor básico sentido } \\
\text { através de uma solução } \\
\text { salina }\end{array}$ & $\begin{array}{l}\text { Fraco: solução salina 0,5\% } \\
\text { Forte: solução salina 1,5\% }\end{array}$ \\
\hline \multicolumn{3}{|c|}{ Textura } \\
\hline Crocância (TCR) & $\begin{array}{c}\text { O que produz ruído seco } \\
\text { e característico ao ser } \\
\text { mordido }\end{array}$ & $\begin{array}{l}\text { Pouca: biscoito tipo Cream Cracker da marca } \\
\text { Tostines }{ }^{\circledR} \text { exposto ao vapor por } 45 \mathrm{~s} \\
\text { Muita: } 60 \mathrm{~g} \text { de Cereal Matinal de milho da marca } \\
\text { Superbom } ®\end{array}$ \\
\hline Oleosidade (TOL) & $\begin{array}{l}\text { Presença perceptível de } \\
\text { óleo na batata frita }\end{array}$ & $\begin{array}{l}\text { Pouca: } 60 \mathrm{~g} \text { de batata frita em } 200 \mathrm{~mL} \text { de óleo de } \\
\text { soja da Marca Sadia }{ }^{\circledR} \text { à temperatura de } 200^{\circ} \mathrm{C} \text { por } \\
4 \mathrm{~min} \\
\text { Muita: } 60 \mathrm{~g} \text { de batata frita em } 200 \mathrm{~mL} \text { de óleo de soja } \\
\text { da Marca Sadia }{ }^{\circledR} \text { à temperatura de } 80^{\circ} \mathrm{C} \text { por } 5 \text { min. } \\
\text { Em seguida, as batatas foram imersas por } 2 \text { min em } \\
\text { óleo de soja frio }\end{array}$ \\
\hline
\end{tabular}

As médias de cada atributo encontradas para as respectivas marcas ( $A, B, C, D, E$ e F) na Análise Quantitativa Descritiva são demonstradas na Tabela 2.

Avaliando os atributos relacionados à aparência das seis marcas de batata palha podese observar que a $\mathrm{F}$ diferiu significativamente $(p<0,05)$ das demais, com valores superiores nos parâmetros de cor amarela, cor queimada e presença de sal na superfície. A intensidade desses atributos leva à depreciação da qualidade dessa marca de batata palha, já que a coloração representa uma das principais características sensoriais desse tipo de produto. Para a espessura do corte da batata palha, as marcas A, B e F apresentaram valores semelhantes não demonstrando diferenças significativas $(p \geq 0,05)$ entre si. A marca $C$ foi a que apresentou os menores valores de espessura do corte de batata em relação às demais.

$\mathrm{Na}$ Figura 3 pode-se observar as maiores semelhanças entre as amostras ( $\mathrm{A}, \mathrm{B}, \mathrm{E}$ e $\mathrm{F}$ ) para o atributo espessura do corte da batata (ECB). Todos os atributos relacionados com a aparência das amostras $C$ e $D$ foram muito semelhantes, conforme demonstra a sobreposição gráfica. As abreviaturas e definições dos termos descritores encontram-se na Tabela 1.

O aroma de produtos fritos assume grande importância sob o ponto de vista sensorial. Dentre as amostras analisadas, a marca $F$ apresentou valores superiores de aroma ranço e queimado, diferindo das demais. As marcas $A, B$ e $E$ não diferiram significativamente $(p \geq 0,05)$ entre si, assim como as marcas C e D.

A representação gráfica da Figura 3 permitiu correlacionar as marcas A, B, C, D, e E em todos os atributos de aroma determinados pela Análise Descritiva Quantitativa (ADQ). No entanto, a marca $\mathrm{F}$ apresentou valores extremamente contrários às demais. 
TABELA 2 - MÉDIAS ${ }^{1}$ DOS TERMOS DESCRITORES² DAS AMOSTRAS DE BATATA-PALHA ENCONTRADAS PARA A ANÁLISE DESCRITIVA QUANTITATIVA

\begin{tabular}{|c|c|c|c|c|c|c|c|}
\hline \multirow[t]{2}{*}{ Atributos } & \multicolumn{7}{|c|}{ Amostras } \\
\hline & A & B & C & D & $\mathbf{E}$ & $\mathbf{F}$ & $\mathrm{DMS}^{3}$ \\
\hline \multicolumn{8}{|c|}{ Aparência } \\
\hline CAM & $2,83^{c}$ & $4,60^{b}$ & $1,47^{d}$ & $1,19^{d}$ & $1,32^{\mathrm{d}}$ & $7,37^{a}$ & 0,3547 \\
\hline CQU & $0,57^{c}$ & $1,81^{b}$ & $0,39^{c}$ & $0,50^{c}$ & $0,60^{c}$ & $7,78^{a}$ & 0,2866 \\
\hline SSU & $2,69^{c}$ & $3,80^{b}$ & $1,01^{\mathrm{e}}$ & $0,87^{e}$ & $2,18^{d}$ & $7,10^{\mathrm{a}}$ & 0,4234 \\
\hline ECB & $6,90^{\mathrm{a}}$ & $6,94^{a}$ & $2,26^{d}$ & $3,38^{c}$ & $5,96^{b}$ & $6,68^{a}$ & 0,3452 \\
\hline \multicolumn{8}{|c|}{ Aroma } \\
\hline ABP & $5,25^{b}$ & $4,88^{b}$ & $3,66^{c}$ & $3,86^{c}$ & $4,62^{b}$ & $6,75^{a}$ & 0,4524 \\
\hline ARN & $0,54^{b}$ & $0,63^{b}$ & $0,12^{c}$ & $0,48^{b}$ & $0,41^{b, c}$ & $2,58^{a}$ & 0,3173 \\
\hline AQU & $0,19^{b}$ & $0,32^{b}$ & $0,10^{b}$ & $0,20^{b}$ & $0,18^{b}$ & $6,64^{a}$ & 0,3083 \\
\hline \multicolumn{8}{|c|}{ Sabor } \\
\hline SBP & $4,89^{b, c}$ & $4,53^{c}$ & $6,05^{a}$ & $5,36^{b}$ & $3,35^{d}$ & $6,08^{a}$ & 0,5076 \\
\hline SRN & $0,40^{b, c}$ & $0,60^{\mathrm{b}}$ & $0,19^{c}$ & $0,20^{c}$ & $0,21^{c}$ & $1,67^{a}$ & 0,2561 \\
\hline SQU & $0,27^{b}$ & $0,33^{b}$ & $0,29^{b}$ & $0,06^{b}$ & $0,18^{b}$ & $5,83^{a}$ & 0,3024 \\
\hline SSA & $2,73^{d}$ & $3,27^{c}$ & $2,76^{d}$ & $3,93^{b}$ & $2,81^{\mathrm{c}, \mathrm{d}}$ & $5,90^{a}$ & 0,505 \\
\hline \multicolumn{8}{|c|}{ Textura } \\
\hline TCR & $6,70^{\mathrm{a}}$ & $2,88^{d}$ & $5,90^{c}$ & $6,54^{a, b}$ & $6,17^{\mathrm{b}, \mathrm{c}}$ & $6,33^{a, b, c}$ & 0,4579 \\
\hline TOL & $6,15^{a}$ & $3,99^{b}$ & $1,74^{\mathrm{d}}$ & $3,74^{\mathrm{b}, \mathrm{c}}$ & $3,61^{c}$ & $6,27^{a}$ & 0,3208 \\
\hline
\end{tabular}

${ }^{1}$ Médias na mesma linha acompanhadas da mesma letra não são significativamente diferentes pelo teste de Tukey $(p \geq 0,05)$. ${ }^{2}$ Descrição dos atributos na Tabela $1 .{ }^{3}$ DMS = Diferença mínima significativa.

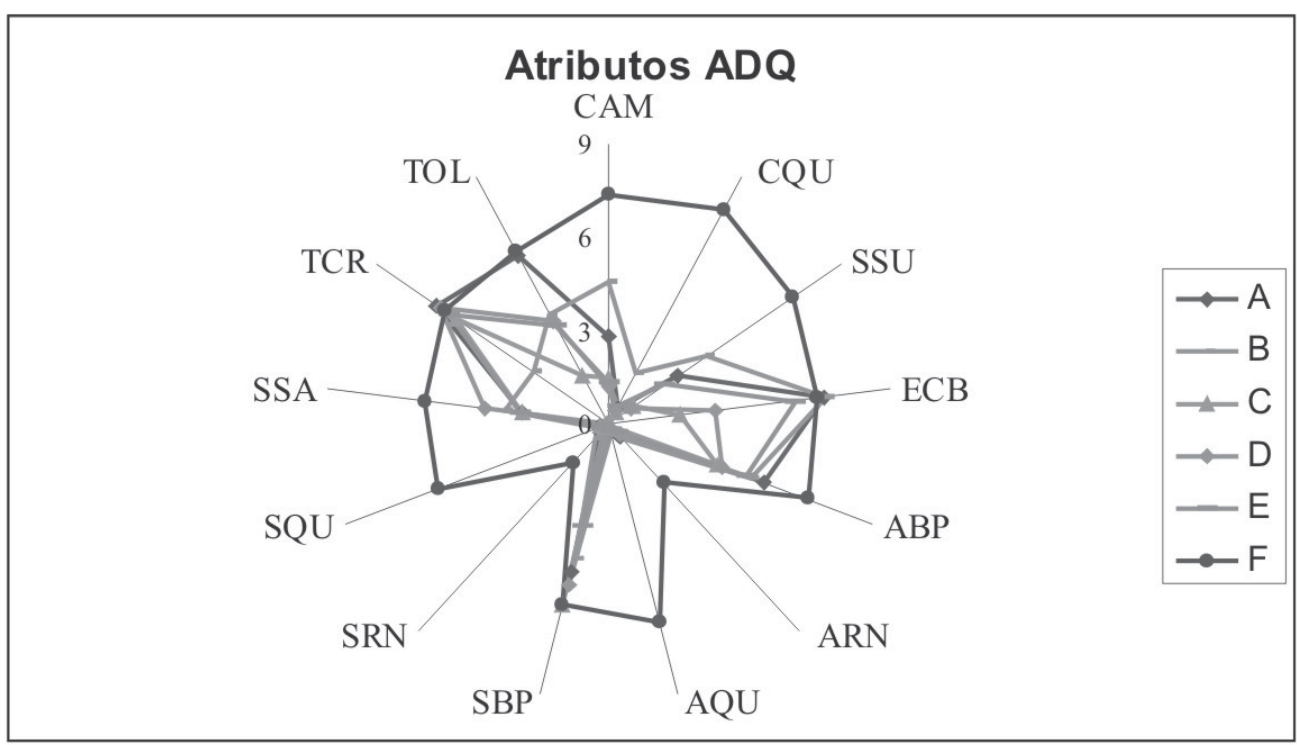

FIGURA 3 - REPRESENTAÇÃO GRÁFICA DOS RESULTADOS DA ANÁLISE QUANTITATIVA DESCRITIVA DE BATATA PALHA PARA TODOS OS

ATRIBUTOS DAS MARCAS A, B, C, D, E e F 
O perfil de sabor das seis marcas de batata palha demonstrou que a marca $\mathrm{F}$ alcançou as maiores médias e diferiu significativamente $(p<0,05)$ das demais quanto aos atributos de sabor de ranço, queimado e salgado. Para o atributo sabor de batata frita, as marcas $\mathrm{C}$ e $\mathrm{F}$ obtiveram médias próximas e não apresentaram diferença significativa $(p \geq 0,05)$.

Comportamento similar para os parâmetros de sabor entre as marcas A, B, C, D e E pode ser observado na Figura 3. A marca $F$ somente apresentou semelhança com as outras marcas quanto ao atributo sabor de batata frita (SBP).

A textura de batata palha é considerada pelos consumidores como um dos principais atributos sensoriais (ALMEIDAet al., 1983). As amostras A, D e F apresentaram o melhor desempenho para o atributo crocância. A amostra $B$ diferiu significativamente $(p<0,05)$ das demais marcas com médias inferiores para esse atributo. As amostras A e $F$ obtiveram valores superiores para o teor de oleosidade, diferindo estatisticamente $(p<0,05)$ das demais. $O$ menor teor de oleosidade foi observado para a amostra $C$.

A marca B (Figura 3) apresentou comportamento diferente das demais quanto ao atributo de crocância das batatas palha (TCR). As marcas B, D e E demonstraram semelhança para o atributo teor de oleosidade (TOL), assim como as marcas A e F.

Na Tabela 3 são apresentados os resultados obtidos pelo teste afetivo de aceitação (teste de consumidor) em relação às características sensoriais de cor, aroma, sabor, textura e impressão global para as marcas de batata palha, submetidos ao teste de Tukey. A análise de variância mostrou diferença significativa $(p<0,05)$ entre as amostras em relação aos atributos cor, sabor e textura, que de modo geral foram os atributos que mais influenciaram os consumidores quanto ao grau de aceitação das amostras.

\section{TABELA 3 - MÉDIAS ${ }^{1}$ DAS NOTAS ATRIBUÍDAS PELOS CONSUMIDORES PARA COR, AROMA, SABOR, TEXTURA E IMPRESSÃO GLOBAL DE BATATA PALHA DAS MARCAS A, B, C, D e E}

\begin{tabular}{cccccc}
\hline MARCAS & COR & AROMA & SABOR & TEXTURA & IMP. GLOBAL \\
\hline A & $6,18^{\mathrm{b}, \mathrm{c} \pm 2,07}$ & $5,36^{\mathrm{a}} \pm 1,99$ & $5,48^{\mathrm{b}} \pm 2,28$ & $6,15^{\mathrm{c}} \pm 2,35$ & $5,60^{\mathrm{b}, \mathrm{c} \pm 2,19}$ \\
B & $6,69^{\mathrm{a}, \mathrm{b}} \pm 1,72$ & $5,39^{\mathrm{a}} \pm 2,10$ & $5,47^{\mathrm{b}} \pm 2,29$ & $5,35^{\mathrm{d}} \pm 2,47$ & $5,30^{\mathrm{c}} \pm 2,12$ \\
C & $5,92^{\mathrm{c}} \pm 2,06$ & $5,31^{\mathrm{a}} \pm 2,00$ & $4,61^{\mathrm{c}} \pm 2,23$ & $6,28^{\mathrm{b}, \mathrm{c}} \pm 1,85$ & $5,23^{\mathrm{c}} \pm 1,94$ \\
D & $7,21^{\mathrm{a}} \pm 1,42$ & $5,99^{\mathrm{a}} \pm 1,81$ & $6,59^{\mathrm{a}} \pm 1,95$ & $7,45^{\mathrm{a}} \pm 1,24$ & $6,83^{\mathrm{a}} \pm 1,65$ \\
E & $6,97^{\mathrm{a}} \pm 1,59$ & $5,64^{\mathrm{a}} \pm 2,00$ & $5,94^{\mathrm{a}, \mathrm{b}} \pm 2,27$ & $6,93^{\mathrm{a}, \mathrm{b}} \pm 1,85$ & $6,29^{\mathrm{a}, \mathrm{b}} \pm 1,75$ \\
F & $2,73^{\mathrm{d}} \pm 1,92$ & $4,25^{\mathrm{b}} \pm 2,10$ & $4,55^{\mathrm{c}} \pm 2,45$ & $5,97^{\mathrm{c}, \mathrm{d}} \pm 2,12$ & $4,18^{\mathrm{d}} \pm 2,24$ \\
\hline
\end{tabular}

${ }^{1}$ Médias \pm Desvio Padrão na mesma coluna acompanhadas da mesma letra não são significativamente diferentes pelo teste de Tukey $(p \geq 0,05)$.

As avaliações da cor das amostras de batata palha demonstraram a preferência do consumidor pelas marcas B, D e E que não apresentaram diferença estatística $(p \geq 0,05)$ entre si. Em relação ao atributo aroma houve semelhança entre todas as marcas, exceto a marca $F$, cuja média $(4,25)$ diferiu significativamente $(p<0,05)$ das demais.

As marcas de batata palha $D$ e $E$ revelaram as maiores médias para os atributos sabor, textura e cor, o que se refletiu em melhores médias para a impressão global. Resultado contrário foi encontrado para a marca $F$, que mereceu a pior aceitação entre os consumidores.

A Figura 4 representa graficamente os resultados obtidos no teste de intenção de compra dos consumidores em relação às seis marcas comerciais de batata palha. Os maiores percentuais 
situados na escala de 5 pontos referente à descrição "certamente compraria" foram encontrados para as marcas D e E. Tal resultado coincide com as maiores notas para o atributo impressão global para as mesmas marcas. Destaca-se ainda o alto índice de rejeição da marca $F$, que recebeu nota 1 de 51\% dos consumidores (certamente não compraria). Esse resultado demonstra o quanto os atributos sensoriais avaliados pelos consumidores são importantes no momento de decisão de compra.

\section{INTENÇÃO DE COMPRA}

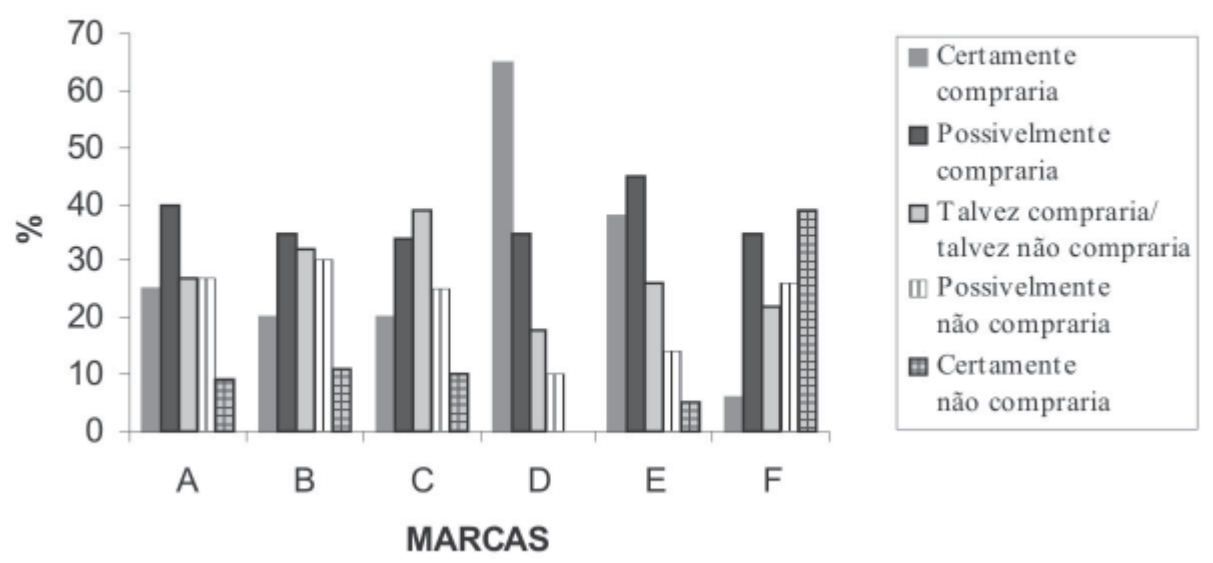

\section{FIGURA 4 - PORCENTAGEM DE INTENÇÃO DE COMPRA PARA AS AMOSTRAS DE BATATA PALHA DAS MARCAS A, B, C, D, E e F}

Mostra-se a representação gráfica da Análise de Componentes Principais (ACP) na Figura 5. A repetibilidade do método foi estudada, uma vez que os julgadores selecionados para a etapa de Análise Descritiva Quantitativa (ADQ) avaliaram as amostras em três repetições. Os resultados de cada amostra de batata palha e suas repetições são representados por três pontos ligados, formando um triângulo. Cada vértice do triângulo corresponde ao ponto de uma das repetições atribuídas pela equipe sensorial. Tal metodologia também foi utilizada por Osawa et al. (2008) para avaliar o perfil sensorial de chá light sabor pêssego; por Tashima e Bolini-Cardello (2003) em estudo com extratos hidrossolúveis de soja, e por Jerônimo, Bolini-Cardello e Serra (2004) em pesquisa sobre o perfil sensorial de aguardentes de cana em função da diluição e variação da acidez da amostra. Obteve-se boa repetibilidade, evidenciada pelo agrupamento dos pontos representativos de cada marca. A distinção entre as marcas e a amostra $F$ pode ser observada pela localização bem definida dessa amostra no gráfico.

Os componentes principais 1 e 2 explicaram juntos $85,85 \%$ da variação entre as marcas. Os atributos descritores são representados pelos vetores, sendo que os mais longos explicam a maior variabilidade entre as marcas mostradas naquele componente principal (eixo 1 e/ou 2). As diferenças entre as marcas de batata palha foram explicadas em $70,50 \%$ pelo componente principal 1 e o vetor descritor cor amarela (CAM) caracterizou esse primeiro eixo. O componente principal 2 obteve baixa explicação $(15,35 \%)$ e a textura crocante (TCR) foi o vetor mais importante nesse eixo.

Na representação gráfica de ACP, as marcas devem se localizar próximas aos vetores que as caracterizam. Como a amostra $\mathrm{F}$ obteve resultados discrepantes em relação às demais, todos os vetores direcionaram-se a ela, exceto o do atributo de textura crocante (TCR). Esses resultados 
concordam com os encontrados na ADQ apresentados na Tabela 2.

A regressão por PLS (Figura 6), entre os atributos sensoriais e a impressão global dos 128 consumidores que realizaram o teste afetivo, evidenciou as características sensoriais dos produtos que contribuem positiva ou negativamente de forma significativa para a aceitação das marcas de batata palha. Assim, os atributos situados acima do eixo X contribuem positivamente para a aceitação do produto, enquanto que os atributos abaixo do eixo contribuem de forma negativa. Verificouse que somente $o$ atributo sabor ranço contribuiu significativa e negativamente para a aceitação das batatas palha avaliadas. Para os demais atributos não foi possível afirmar se os mesmos contribuem de forma negativa ou positiva devido similaridade entre as amostras avaliadas, exceto a marca F.

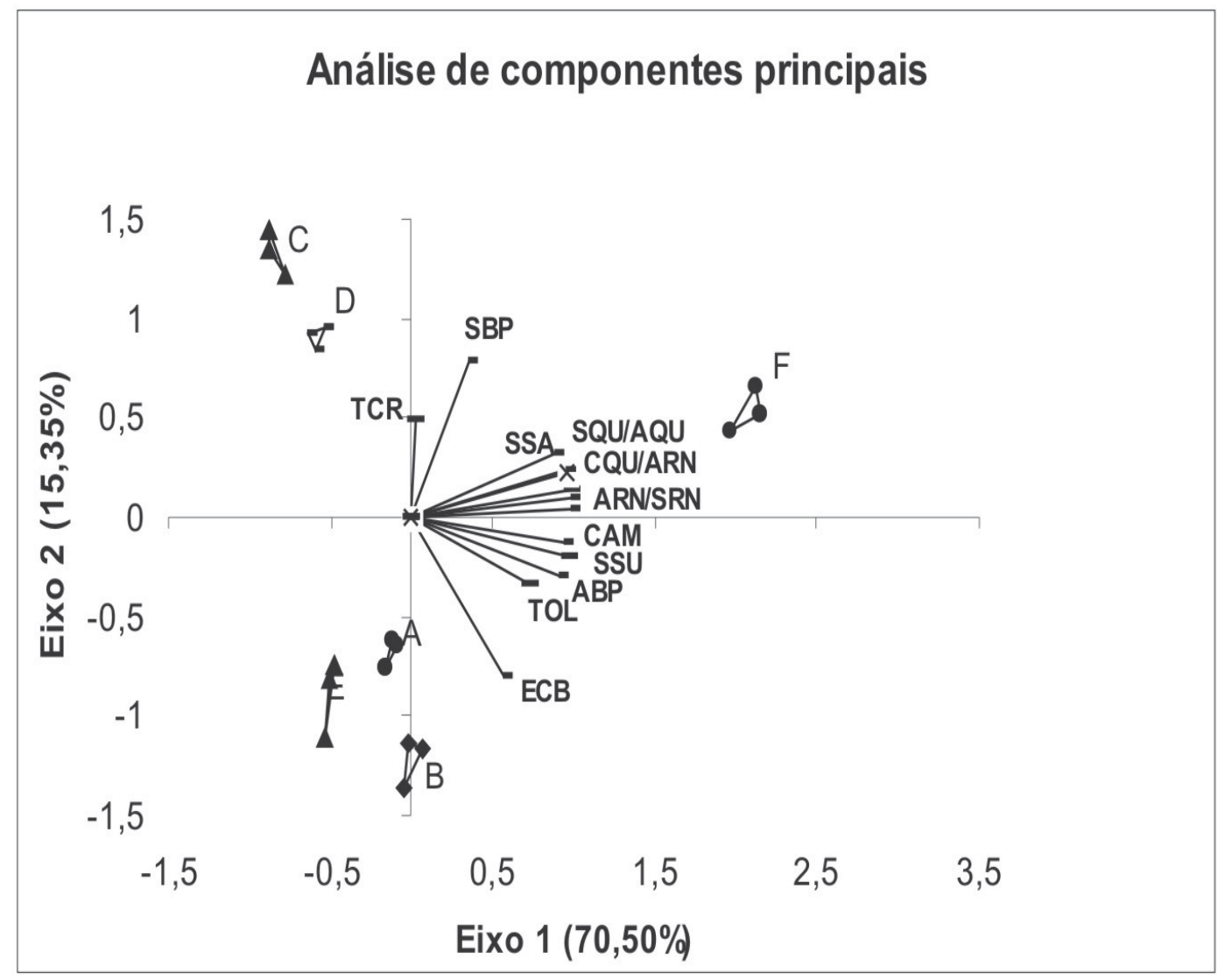

\section{FIGURA 5 - PROJEÇÃO BIDIMENSIONAL DA ANÁLISE DE COMPONENTES PRINCIPAIS DOS TERMOS DESCRITORES DE BATATA PALHA DAS \\ MARCAS A, B, C, D, E e F}

A Figura 7 apresenta o mapa da correlação da regressão PLS da impressão global dos consumidores e características das amostras. A concentração dos pontos que representam os termos descritores junto à amostra $F$ confirma suas maiores médias em relação aos atributos avaliados. A localização das amostras A, D e E, do lado oposto do gráfico e próximas ao atributo impressão global, demonstra que essas marcas alcançaram maior aceitação pelo consumidor e apresentaram menor intensidade na maioria dos termos descritores avaliados. A proximidade do atributo crocância no gráfico em relação à impressão global evidencia a preferência do consumidor por batatas palha com maior crocância. Dessa forma, o PLS constitui método eficaz na determinação das características importantes para os produtos e mostra boa capacidade preditiva (PEDRO, 2004). 
por batatas palha com maior crocância. Dessa forma, o PLS constitui método eficaz na determinação das características importantes para os produtos e mostra boa capacidade preditiva (PEDRO, 2004).

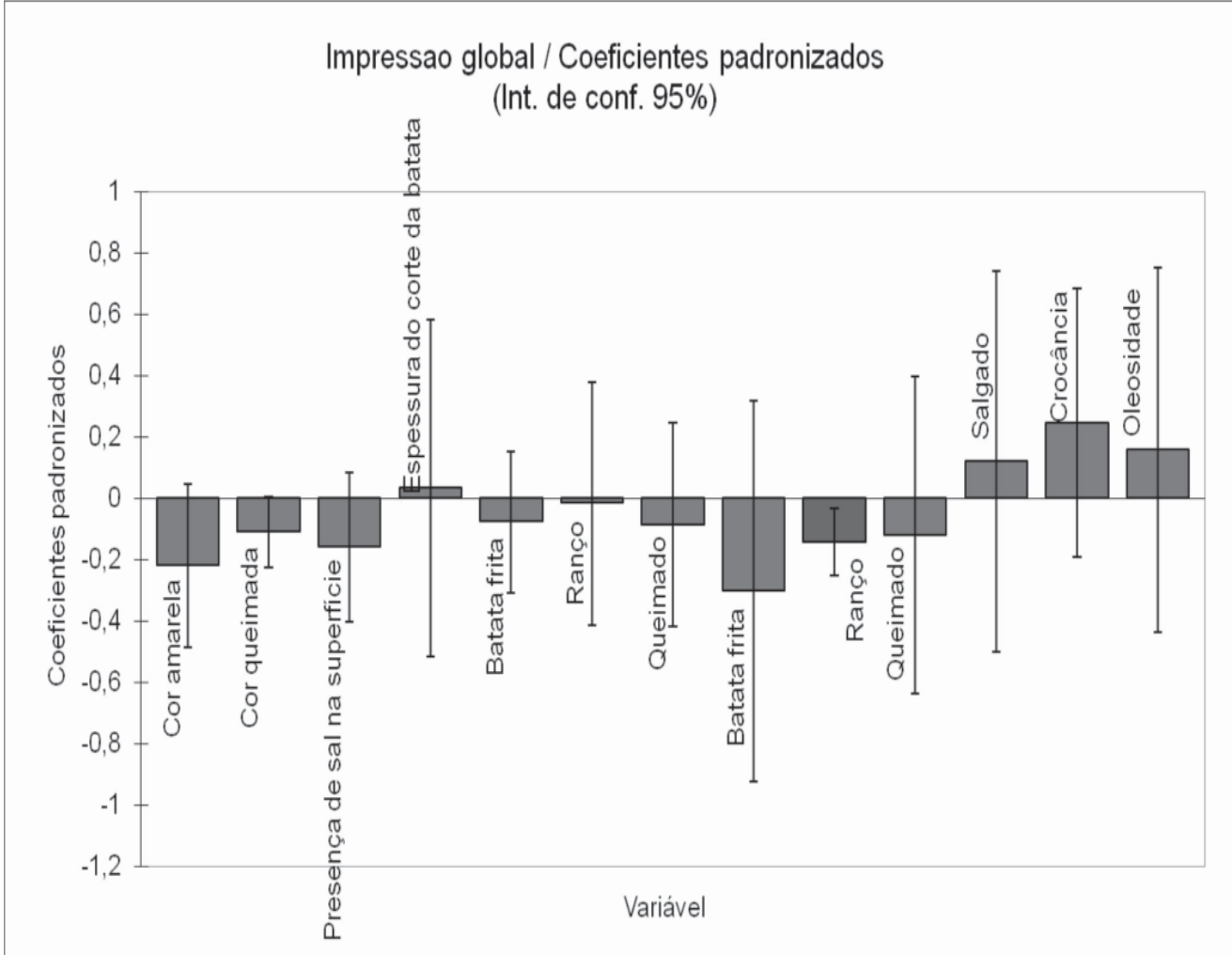

FIGURA 6 - COEFICIENTES PADRONIZADOS DA REGRESSÃO PLS DOS ATRIBUTOS SENSORIAIS E IMPRESSÃO GLOBAL DAS BATATAS PALHA DAS MARCAS A, B, C, D e E

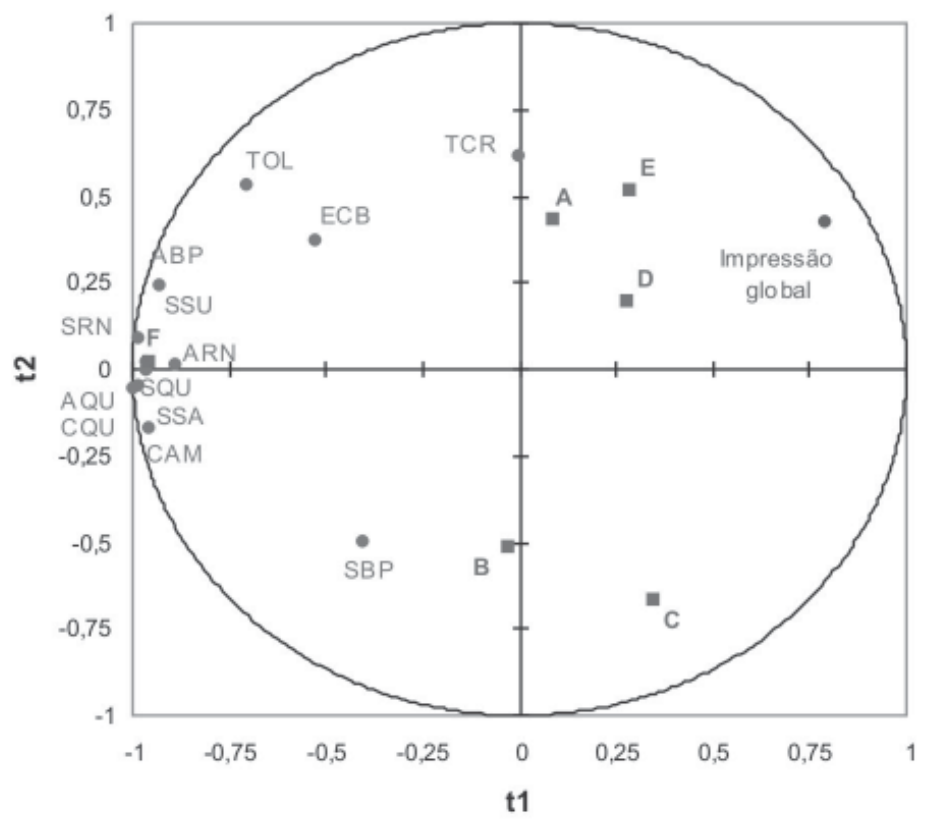

FIGURA 7 - MAPA DA CORRELAÇÃO PLS ENTRE AS AMOSTRAS, CARACTERÍSTICAS SENSORIAIS E IMPRESSÃO GLOBAL

Nota: Descrição dos atributos na Tabela 1. 


\section{CONCLUSÃO}

A análise descritiva quantitativa permitiu o levantamento e a avaliação de vários aspectos importantes da batata palha. Dentre as seis marcas comerciais avaliadas, a F destacou-se pela maior intensidade de vários termos descritores, percebida pela equipe sensorial, o que contribuiu para a depreciação de sua qualidade. Tal fato se confirmou com a menor aceitação dessa marca pelos consumidores nos testes afetivos.

As marcas D e E destacaram-se como as de melhor aceitação pelos consumidores. O teste afetivo de aceitação demonstrou que os atributos cor, sabor e textura foram os mais significativos para os resultados. Já no mapa de correlação PLS destacou-se o atributo textura, indicando maior influência da crocância na impressão global das batatas palha. A análise de componentes principais mostrou correlação com os demais testes, evidenciando as diferenças da amostra $\mathrm{F}$ das outras marcas em relação a todos os atributos sensoriais estudados.

As técnicas empregadas na análise sensorial das marcas de batata-palha possibilitaram a definição de atributos sensoriais importantes e ainda auxiliaram na obtenção de visão preditiva de mercado de produto de consumo em ascensão por meio dos resultados do perfil sensorial descritivo e dos testes afetivos de aceitação.

\section{ABSTRACT}

\section{EVALUATION OF THE SENSORY PROFILE AND CONSUMER TEST OF STICK POTATO}

The aim of this study was to determine the sensory profile, acceptance and purchase intention of six brands of traditional potato sticks, named A, B, C, D, E and F, acquired from supermarkets in the region of Campinas, SP (BRAZIL). The Quantitative Descriptive Analysis (QDA) determined thirteen descriptors: yellow color, burnt color, presence of salt on the surface, slice thickness, chips aroma, burnt aroma and rancidity aroma, salty flavor, burnt flavor, rancidity flavor and chips flavor, texture crunchy and oily. ANOVA results of affective test showed that attributes color, flavor and texture were of great influence over consumers, and the highest average rates corresponded to brands D and E. Brand F differed significantly $(p<0.05)$ when compared to other brands, with higher values in the parameters yellow color, burnt color, rancidity flavor and burnt flavor. The main components 1 , yellow color and 2, crunchy texture together explained $85.85 \%$ of the variation between brands. On the map of the PLS regression correlation of the overall impression of consumers and characteristics of the samples emphasized the proximity of the crunchy attribute in the graphic toward overall impression which indicates the consumer preference for more crunchy potato sticks.

KEY-WORDS: STICK POTATO; SENSORIAL DESCRIPTIVE PROFILE; AFFECTIVE TEST.

\section{REFERÊNCIAS}

1 ABBA. Associação Brasileira da Batata. Variedades, 2009. Disponível em: http://www.abbabatatabrasileira.com.br/ images/variedades/asterix.pdf. Acesso em: junho/ 2009.

2 ALMEIDA, L.A.; GASPARINO FILHO, J.; PASCHOALINO, J.E.; BERNHARDT, L.W.; CANTO, W.L. Batata pré-frita e hortaliças congeladas. Campinas: ITAL, 1983. $91 \mathrm{p}$.

3 ARRUDA, C.R. Análise das etapas do processamento de batata chips. 2004. 46 p. Trabalho de conclusão de curso (Graduação em Engenharia de Alimentos), Universidade Católica de Goiás, Goiânia, 2004.

4 CAPEZIO, S.; HUARTE, M.; CARROZZI, L. Selección por peso específico en generaciones tempranas en el mejoramiento de la papa. Revista Latinoamericana de la Papa, v. 5/6, p. 54-63, 1993.

5 COELHO, A.H.R. ; VILELA, E.R. ; CHAGAS, S.J.R. Qualidade de batata (Solanum tuberosum L.) para fritura, em função dos níveis de açúcares redutores e amido, durante o armazenamento refrigerado e à temperatura ambiente com atmosfera modificada. Ciência e Agrotecnologia, v.23, n.4, p.899-910, 1999.

6 DAMÁSIO, M. H.; COSTEL, E. Análisis sensorial descriptivo: generación de descriptores y selección de catadores. Revista Agroquímica y Tecnologia de Alimentos, v.31, n.2, p.165-178, 1991. 
7 FREITAS, S.T.; BISOGNIN, D.A.; GÓMEZ, A.C.S.; SAUTTER, C.K.; COSTA, L.C.; RAMPELOTTO, M.V. Qualidade para processamento de clones de batata cultivados durante a primavera e outono no Rio Grande do Sul. Ciência Rural, v. 36, n.1, p. 80-85, 2006.

8 JERONIMO, E.; BOLINI-CARDELLO, H.M.A.; SERRA, G.E. Perfil sensorial de aguardente de cana em função da diluição e variação da acidez da amostra. Boletim do CEPPA, v. 22, n. 1, p. 51-64, 2004.

9 MEILGAARD, M.; CIVILLE, G.V.; CARR, B.T. Sensory evaluation techniques. $4^{\text {th }}$ ed. Boca Raton: CRC Press, 2006. $281 \mathrm{p}$.

10 MURRAY,J.M.; DELAHUNTY,C.M.;BAXTER,I.A. Descriptive sensory analysis: past, present and future. Food Research International, v.34, p.461-471,2001.

11 PEDRO, A. M. K. Determinação simultânea e não-destrutiva de sólidos totais e solúveis, licopeno e beta-caroteno em produtos de tomate por espectroscopia no infravermelho próximo utilizando calibração multivariada. 2004. 60 p. Dissertação (Mestrado em Química), Universidade Estadual de Campinas, Campinas, 2004.

12 REIS, F. R. Efeito dos processos de branqueamento e acidificação sobre a cor e a absorção de gorduras de batatas-palha. 2007. 52 p. Dissertação (Mestrado em Tecnologia de Alimentos), Universidade Federal do Paraná, Curitiba, 2007.

13 RODRIGUES, N.S.S. Avaliação tecnológica e sensorial de novos genótipos de batata (Solanum tuberosum, L.) para industrialização na forma de pré-fritas e congeladas. 1990. 198 p. Dissertação (Mestrado em Engenharia de Alimentos), Faculdade de Engenharia de Alimentos, Universidade Estadual de Campinas, Campinas, 1990.

14 SAS INSTITUTE. SAS users' guide: statistics. $2^{\text {nd }}$ ed. Cary, 2003.

15 STONE, H.; BLEIBAUM, R.N.; THOMAS, H.A. Sensory evaluation practices. $4^{\text {th }}$ ed. New York: Academic Press, 2012. $438 \mathrm{p}$.

16 TASHINA, E.H.; BOLINI-CARDELLO, H.M.A. Perfil sensorial de extrato hidrossolúvel de soja (Glicine max L. Merril) comercial adoçado com sacarose e com sucralose. Boletim do CEPPA, Curitiba, v. 21, n. 2, p. 409-428, 2003.

17 TFOUNI, S.A.V.; MACHADO, R.M.D.; GARCIA, L.C.; AGUIRRE, J.M.; GASPARINO FILHO, J. Batata chips e palha. Campinas: ITAL, 2003. 73 p. (Agronegócio, 3).

18 XLSTAT-PRO: user's guide. New York: Addinsoft Inst., 2005. Version 7.5.3.

19 ZORZELLA, C.A.; VENDRUSCOLO, J.L.S.; TREPTOW, R.O.; ALMEIDA, T.L. Caracterização física, química e sensorial de genótipos de batata processados na forma de chips. Brazilian Journal of Food Technology, n.1, v.6, p.15-24, 2003. 